\title{
Polymer Chain Dynamics: Evidence of Nonexponential Mode Relaxation Using Thermally Stimulated Depolarization Current Techniques
}

\author{
S. Arrese-Igor, ${ }^{1}$ A. Alegría, ${ }^{1,2}$ and J. Colmenero ${ }^{1,2,3}$ \\ ${ }^{1}$ Centro Física de Materiales (MPC), Centro Mixto CSIC-UPV/EHU, Paseo Manuel Lardizabal 5, 20018 San Sebastián, Spain \\ ${ }^{2}$ Departamento de Física de Materiales UPV/EHU, Apartado 1072, 20080 San Sebastián, Spain \\ ${ }^{3}$ Donostia International Physics Center, Paseo Manuel Lardizabal 4, 20018 San Sebastián, Spain
}

(Received 28 March 2014; published 14 August 2014)

\begin{abstract}
The slowest $(p=1)$ mode relaxation of several polyisoprenes has been experimentally isolated by thermally stimulated depolarization current techniques. Close to the glass transition the $p=1$ mode deviates from the exponential behavior assumed by Rouse and tube-reptation theories. This effect is found to be a consequence of the closeness of $\tau_{p=1}$ and $\alpha$-relaxation time scales. The scenario resembles that of broadened fast component dynamics in polymer blends with high dynamic asymmetry and suggests a possible general interpretation in terms of the effect of local density fluctuations ( $\alpha$ relaxation) on chain dynamics.
\end{abstract}

DOI: 10.1103/PhysRevLett.113.078302

PACS numbers: 82.35.Lr, 61.25.H-, 64.70.pj, 65.60.+a

Fluctuations of the end-to-end vector of a polymer chain governing the global chain dynamics are at the basis of viscoelastic properties. The most basic model for chain dynamics, the Rouse model [1], pictures a linear polymer chain as a series of beads and springs subjected to random forces in a medium with constant friction representing the intermolecular interactions exerted by the surrounding chains. The correlation function for the end-to-end vector $\vec{R}(t)$ is given by the so-called Rouse-modes correlators (Rouse modes in the following),

$$
\langle\vec{R}(t) \cdot \vec{R}(0)\rangle \propto \sum_{p: \text { odd }}^{N-1} \cot ^{2}\left(\frac{p \pi}{2 N}\right) \exp \left(-\frac{t}{\tau_{p}}\right),
$$

where $N$ is the number of beads and $\tau_{p}$ is the relaxation time associated with the $p$ th Rouse mode, which scales as $\tau_{p}=\tau_{p=1} / p^{2}$ in the relevant low- $p$ range. Equation (1) shows that only the odd Rouse modes contribute to the end-to-end vector correlation function. Additionally, the contribution from the high- $p$ modes is strongly suppressed due to the amplitude factor, which for low $p / N$ ratios approximately behaves as $1 / p^{2}$, and, therefore, the end-toend correlation function is dominated by the slow (low- $p$ ) Rouse modes. Although the Rouse approach obviously fails in describing the melt dynamics of long chains, the above equation for the end-to-end vector also applies for entangled polymers within the reptation theory $[2,3]$ and its extensions [4] with a different physical meaning for $\tau_{p=1}$. We note that the above expression for the correlation function of the end-to-end vector would still remain formally valid in the case of nonexponential relaxation of the modes provided these were orthogonal. The orthogonal and exponential nature of modes arises as a consequence of neglecting, respectively, the spatial and time correlations of the random forces acting on a polymer segment.
From an experimental point of view it is very difficult to access the response of a single mode, and generally exponential modes are assumed, i.e., time correlations between forces are neglected, particularly in the hightemperature range where the relaxation functions decay relatively fast and are experimentally accessible. However, molecular dynamics simulations usually show deviations from the exponential behavior even in the low- $p$ range [5-8]. Here we present for the first time an explanation of how the relaxation of the $p=1$ mode can be isolated by means of the thermally stimulated depolarization current (TSDC) technique. Moreover, the very low frequency range naturally covered by TSDC allows investigation of chain dynamics for different molecular weights at relatively low temperatures approaching the range of the kinetic glass transition $\left(T_{g}\right)$ where a polymer melt ultimately becomes a glass. This is a range where end-to-end fluctuations were never explored in detail before. We show that in this range the $p=1$ mode significantly deviates from the exponential behavior. This effect is found to be a consequence of the closeness in the time scales of the chain dynamics $\left(\tau_{p=1}\right)$ and of the so-called structural $\alpha$ relaxation $\left(\tau_{\alpha}\right)$. This relaxation is a universal dynamic feature of glass-forming systems directly related to the glass-transition process, and it is driven by the time evolution of the local density fluctuations in the system at intermolecular scale. The scenario resembles that of polymer blends with high dynamic asymmetry where the chain dynamics of the fast component broadens when approaching the $\alpha$ relaxation of the slow component $[6,8,9]$, and suggests a possible general interpretation in terms of the effect of local density fluctuations on chain dynamics.

In a TSDC experiment one measures the thermally stimulated release of polarization which was frozen in during a previous electrical field poling [10,11]. A basic TSDC experiment includes two main steps. First, the 
sample material is polarized in a dc field at high temperature $T_{\mathrm{on}}$, where particular dipolar units are free to move and then is cooled down with the field on in order to freeze in the sample polarization. In a second step, at low temperature the field is switched off, and during a subsequent linear heating the electric current due to the thermally stimulated release of the polarization is recorded as a function of temperature. A peak in the electric current is obtained when the molecular mobility becomes high enough to randomize dipolar orientation. The global TSDC experiment described above contains contributions from all dielectrically active relaxations. Alternatively, by using partial polarization methods, TSDC spectra enhancing some part of the sample response can be measured. In this case, during cooling the polarizing field is removed at a certain temperature $T_{\text {off }}$, so that polarization contributions which are able to fully relax at $T<T_{\text {off }}$ do not contribute to the spectrum during the subsequent heating, and only the relaxation of slower components produces the measured depolarization current.

Partial polarization methods have often been applied to the study of polymer dynamics [10,12-14]. Results obtained by these techniques, however, can be difficult to interpret [15] and give rise to nonphysical results when the basic assumption of the method (the existence of a set of discrete elementary contributions) is not fulfilled. In contrast, partial polarization TSDC is very well suited to the study of polymer chain dynamics because the decomposition of the chain relaxation in several components (modes) is well defined as reflected in the expression of the end-to-end vector correlation function in Eq. (1). Therefore, in this case partial polarization can be used to isolate different components of the chain relaxation in general and the most intense and slowest one, $p=1$, in particular. So, having direct experimental access to the slowest relaxation mode opens a new way to test the accuracy of different theoretical models, which in general provide specific predictions for this component.

Polyisoprene (PI) is an A-type polymer in the Stockmayer classification. It contains dipole moments along the chain backbone that do not cancel at the whole chain, giving rise to an "end-to-end" net polarization vector whose fluctuations can be followed by dielectric relaxation. PI is a very well studied polymer in general and was the object of many investigations regarding chain dynamics by broadband dielectric spectroscopy (BDS) in particular [16-18]. Here we have studied by TSDC a series of monodisperse PIs in a wide range of molecular weights. The dielectric and thermal response of these samples was previously characterized by BDS and differential scanning calorimetry. Partial polarization experiments were performed by TSDC at $3 \mathrm{~K} / \mathrm{min}$ heating rate and variable $T_{\text {off }}$.

Figure 1 shows the result of several partial polarization experiments in PI82 (molecular weight $M_{w}=82 \mathrm{kDa}$ ) with variable $T_{\text {off }}$. As $T_{\text {off }}$ increases, the low-temperature wing of the curve extends less towards low temperatures while the high-temperature flank stays invariable. This is because, as $T_{\text {off }}$ increases, a larger fraction of fast components no longer contribute to the TSDC current until eventually above certain $T_{\text {off }}$ only the contribution of the slowest $p=1$ component remains. The fact that the curves do not continue narrowing above a certain $T_{\text {off }}$ value is indicative that we have successfully isolated the slowest component [19].

The analysis of depolarization current curves other than the mere identification of the peak position requires the assumption of some model for the relaxation of the polarization. In the simplest case of a single exponential relaxation, the instantaneous value of the stored polarization charge and depolarization current relate as $i(t)=-d Q / d t=Q(t) / \tau$, where $\tau$ is the relaxation time. Since the depolarization process in a TSDC experiment is obtained in a constant rate $(q)$ heating run, $\tau(T)$ can be calculated as a function of temperature directly from TSDC experimental data by the so-called Bucci and Fieschi equation [20]

$$
\tau(T)=\frac{-\frac{1}{q} \int_{T}^{T_{f}} i\left(T^{\prime}\right) d T^{\prime}}{i(T)}
$$

where $T_{f}$ is a temperature where the polarization is completely lost. Figure 2 shows by thick lines the characteristic times so obtained for the $p=1$ mode for relatively long PI chains $\left(M_{w} \gtrsim M_{e}\right.$, where $M_{e}$ is the entanglement molecular weight). Previous studies of PI chain motion by BDS measurements in the $10^{-1}-10^{6} \mathrm{~Hz}$ frequency range concluded that the $T$ dependence of the normal mode was the same for high molecular masses around and above $M_{e}$, following a "universal" WilliamsLandel-Ferry (WLF) law [21]. As shown in Fig. 2, the agreement with the extrapolation of the values measured by BDS (crosses denote measured points, dashed lines denote "universal" WLF fits) is very good in all cases, corroborating the suitability of the mentioned universal WLF also for this nonconventional time or frequency range.

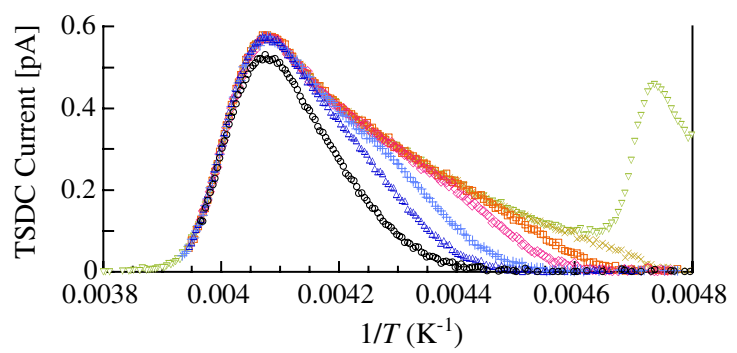

FIG. 1 (color online). Depolarization current curves for partial polarization measurements of PI82 at variable $T_{\text {off }}(239,238$, $237,232,228,222,218,213$, and $173 \mathrm{~K}$ from left to right). 


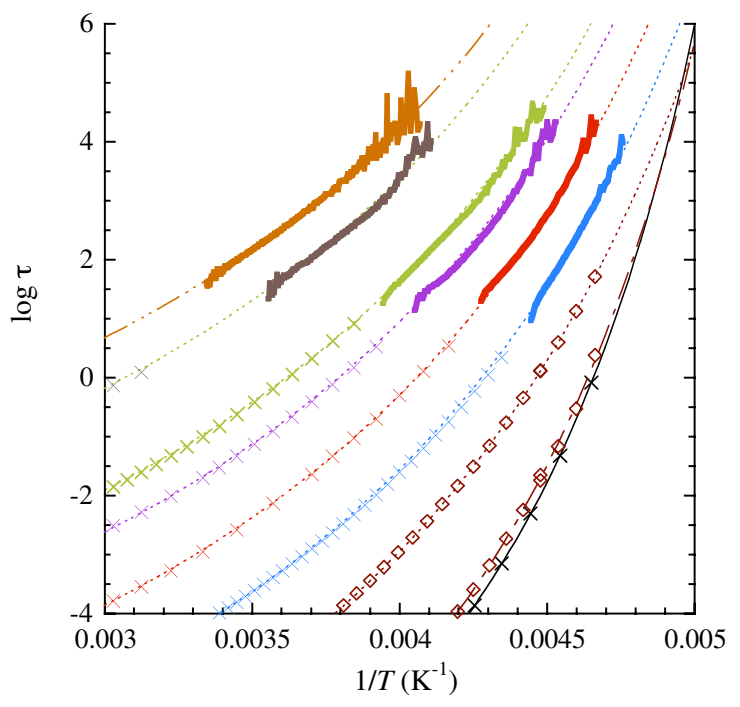

FIG. 2 (color online). From left to right: Times for chain relaxation of PI400 $\left(M_{w}=400 \mathrm{kDa}\right), \operatorname{PI} 320\left(M_{w}=320.2 \mathrm{kDa}\right)$, PI82 $\quad\left(M_{w}=82 \mathrm{kDa}\right), \quad$ PI53 $\quad\left(M_{w}=53 \mathrm{kDa}\right), \quad$ PI19 $\left(M_{w}=19 \mathrm{kDa}\right)$, PI10 $\left(M_{w}=10.5 \mathrm{kDa}\right)$, PI1 $\left(M_{w}=1.2 \mathrm{kDa}\right)$, and $\alpha$ relaxation of PI1 and PI82. Data for PI1 were shifted -0.0004 in the $x$ axis. Thick lines: times by TSDC curve analysis. Crosses $\times(\diamond$ for PI1): times by BDS. Thin lines: fits of BDS times to a WLF (chain) or Vogel-Fulcher-Tammann equation [21] ( $\alpha$ relaxation; dashed line for PI1 and solid line for PI82).

The above results (samples with $M_{w} \gtrsim M_{e}$ ) strongly support the exponential character of the modes assumed by Rouse and reptation models, and no trace of the deviations from the exponential behavior mentioned in the introduction have been observed so far. As noted previously, the exponential nature of modes arise as a consequence of neglecting time correlations of the random forces acting on a polymer segment. It is noteworthy that the Rouse model assumes a polymer chain to be in a Markovian bath, i.e., that all dynamical correlations in the system are much faster than the single chain dynamics. In the high-temperature range where the characteristic time of the $\alpha$ process $\tau_{\alpha}$ is usually well separated from that of the slowest chain mode $\left(\tau_{p=1}\right)$, the Markovian assumption is a good approximation. However, this could not be the case for scenarios where the chain and structural $(\alpha)$ relaxations have close time scales. For the relatively high molecular weight samples considered thus far, we realize that the ratio between $\tau_{p=1}$ and $\tau_{\alpha}$ is at least 3 orders of magnitude, which might be too large for the emergence of nontrivial effects related to the closeness of the $\alpha$ relaxation. On decreasing $M_{w}$, in contrast, the ratio between $\tau_{p=1}$ and $\tau_{\alpha}$ progressively decreases (see data for PI1 included in Fig. 2) and the general assumption of uncorrelation of random forces in time might not be guaranteed leading to nonexponential behavior.

With these ideas in mind we have extended the TSDC measurements to lower $M_{w}$ PIs, namely, PI5 ( $M_{w}=$ $5.3 \mathrm{kDa})$, PI $4 \quad\left(M_{w}=4.5 \mathrm{kDa}\right)$, PI3 $\left(M_{w}=2.9 \mathrm{kDa}\right)$, and PI1. The exponential or nonexponential nature of the isolated $p=1$ component was tested by comparison with theoretically calculated curves. The model depolarization current curves were calculated making use of (i) the reduced time formalism [22], which allows calculating the temperature dependence of the stored charge as [15]

$$
Q(T)=Q_{0} \exp \left[-\frac{1}{q}\left(\int_{T i}^{T} \frac{d T^{\prime}}{\tau\left(T^{\prime}\right)}\right)^{\beta}\right]
$$

where $\beta$ stands for the shape parameter of a KohlrauschWilliams-Watts $[23,24]$ function describing the relaxation of nonexponential processes in general and $T_{i}$ is the initial temperature of the heating ramp, (ii) a Rouse-like model for the chain motion where we allow the modes to be nonexponential, and (iii) the superposition principle [25] for the calculation of partial polarization currents [15].

Figure 3 shows by symbols the depolarization current curves for the $p=1$ mode obtained by partial polarization experiments in PI10, PI5, PI4, PI3, and PI1. In the same figure dotted lines represent the model currents calculated with $\beta=1$ (exponential Rouse model) [26]. This model satisfactorily describes measured depolarization current for PI10 within experimental uncertainty. However, as molecular weight decreases, the exponential character becomes more and more compromised and the experimental curves are systematically broader than the expectation under an exponential behavior. For the PI1 sample the exponential model does not even reproduce peak position and it is clearly narrower in any case. This mismatch in the peak position is not due to inconsistency between the WLF model law times and TSDC experimental results, but a consequence of assuming an exponential model. Calculations with $\beta=0.66$ notably improve the agreement with experimental results reproducing peak position. The stretching parameter $\beta$ of the $p=1$ mode for PI10, PI5, PI4, PI3, and PI1 was determined by generating model partial polarization current curves with various $\beta$ values (see solid lines in Fig. 3). Obtained values are plotted with squares in Fig. 4 as a function of $\log \left(\tau_{p=1} / \tau_{\alpha}\right)$ at the temperature of the TSDC depolarization current maxima for different $M_{w}$. Results show that there is an increase of the nonexponential character as $\tau_{p=1}$ and $\tau_{\alpha}$ times approach each other. The observed behavior is consistent with the proposed scenario where exponentiality would be lost as a consequence of the effect of local density fluctuations $(\alpha$ process) breaking the condition of uncorrelated random forces in time.

Broad global chain relaxations (in comparison with the expectation for exponential relaxation of the Rouse modes) have also been experimentally observed in an, in principle, different context, particularly that of the chain dynamics of the fast component of polymer blends with high dynamic asymmetry [9]. Nonexponential Rouse modes' relaxation has also been deduced even for the low- $p$ range from molecular dynamics simulation in such systems $[6,8]$. This 


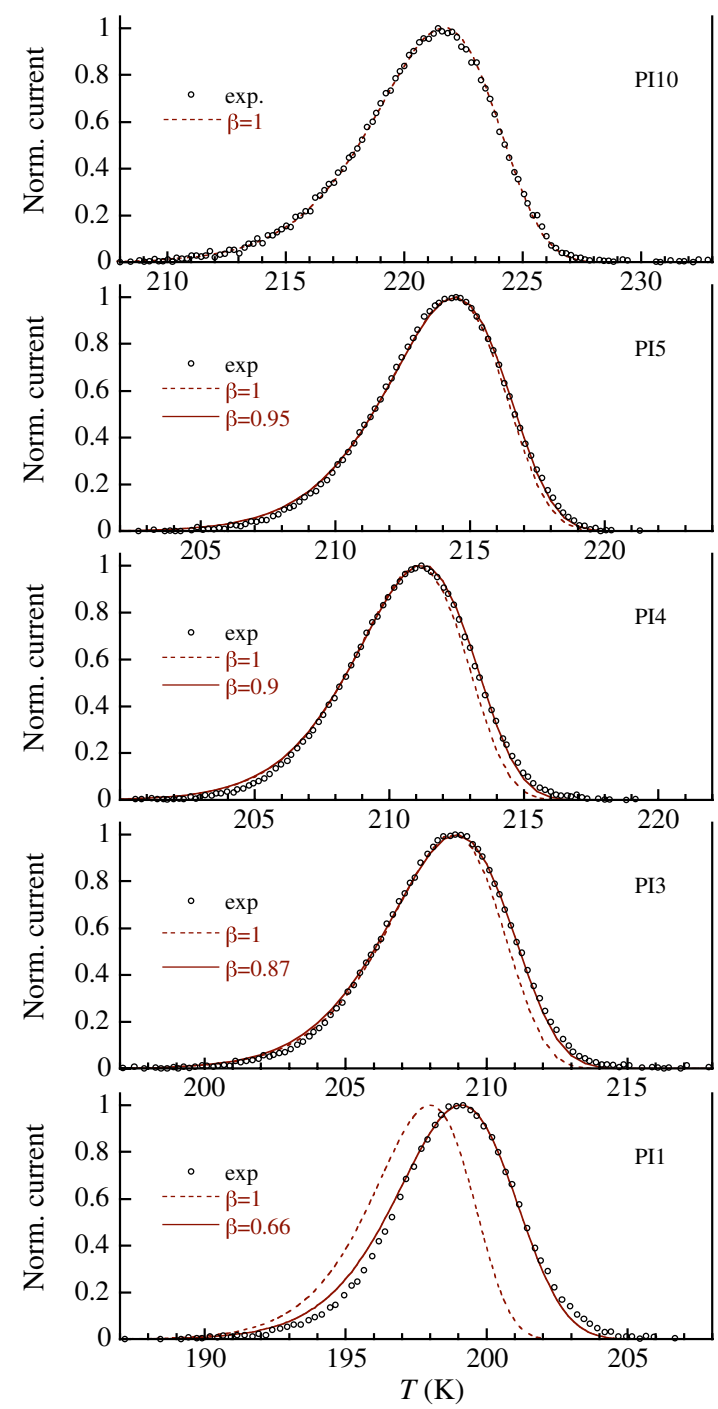

FIG. 3 (color online). Depolarization current curves. Dashed lines and solid lines represent $\beta=1$ and $\beta \neq 1$ model curves, respectively.

behavior was attributed to the effect of the slowing down of the $\alpha$ relaxation of the slow component of the blend $[6,9,27]$. Both situations (blends and homopolymers) could be enclosed within the same scenario, taking into account that in the case of the blend the local density fluctuations affecting the chain dynamics of the fast component are driven by the $\alpha$ relaxation of the slow component. Thus, the relevant parameter would still be $\tau_{p=1} / \tau_{\alpha}$, but now $\tau_{\alpha}$ is the $\alpha$-relaxation time of the slow component in the blend (and $\tau_{p=1}$ is that of the $p=1$ mode for the fast component). The equivalence of these two scenarios was tested by measuring by TSDC the chain dynamics of PI as the fast component of two well-known asymmetric blends: PI-polyvinylehtylene (PVE) and PI-polytertbutylstyrene (PtBS). As in the case of PI homopolymers, the $\beta$ parameter of the $p=1$ mode of PI in the blends was determined from partial polarization TSDC measurements. Results for PI-PVE blends at the two

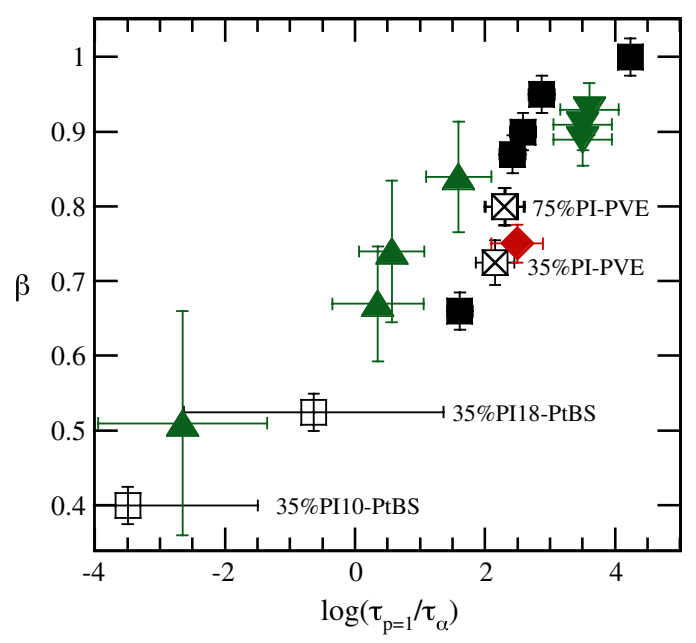

FIG. 4 (color online). $\quad \beta$ parameter for the $p=1$ mode of PI ( $\mathbf{\square})$, PPG $(\diamond)$, and PEO $(\boldsymbol{\nabla})$ homopolymers, PI10 in PI10-PVE blends $(\otimes)$, PI18 and PI10 in PtBS blends $(\boxplus)$, and PEO in PMMA blend $(\boldsymbol{\Lambda}, 20 \%$ wt of PEO).

indicated compositions are included in Fig. 4 (boxed $x$ symbol). As it can be seen, these $\beta$ values nicely match the homopolymers' behavior. $\beta$ is close for homopolymers and blends when compared at equivalent $\tau_{p=1} / \tau_{\alpha}$, with $\tau_{\alpha}$ the characteristic time of the relevant local density fluctuations ( $\alpha$ relaxation of PI for the homopolymers and $\alpha$ relaxation of the slow component PVE for the blends). Because of the strong dynamic difference between the components of asymmetric blends, for these we can face situations where $\tau_{p=1} / \tau_{\alpha} \lesssim 1$ [9], something that is not possible in homopolymers. The two points for PI-PtBS blends included in Fig. 4 (boxed plus symbol) actually correspond to such situation. In this case, the large uncertainty in the $x$ axis reflects the experimental difficulties for determining the $\alpha$ relaxation time for PtBS in the blend [9]. Despite this uncertainty, PI-PtBS data seem to follow the trend of PI and PI-PVE data. In order to stress the generality of theses ideas, we have also included in Fig. 4 a data point corresponding to another type-A polymer [polypropylene glycol (PPG) $M_{w}=4.2 \mathrm{kDa}$, which nicely agrees with the PI data trend. Moreover, we have also considered data obtained [28] from previously published molecular dynamics simulations corresponding to poly(ethylene oxide) (PEO) [29] and PEO in a blend with poly(methyl methacrylate) (PMMA) [8]. Within the large error bars, PEO data also seem to follow the trend observed for PI data, giving support to the general interpretation proposed here.

In summary, we have demonstrated that the relaxation of the slowest chain-dynamics mode $(p=1)$ can experimentally be isolated by taking advantage of the TSDC capabilities. We have found that this relaxation deviates notably from the exponential decay, i.e., from the behavior assumed by Rouse and tube-reptation theories, as soon as the characteristic time of the local density fluctuations in the system $\tau_{\alpha}$ approaches $\tau_{p=1}$. This scenario resembles that of 
polymer blends with dynamic asymmetry and suggests a possible general interpretation.

We acknowledge the support from the following research projects: MAT2012-31088, supported by the Spanish Ministry "Ministerio de Economía y Competitividad," and IT-654-13, supported by the Basque Government.

[1] E. P. Rouse, J. Chem. Phys. 21, 1272 (1953).

[2] P. G. de Gennes, J. Chem. Phys. 55, 572 (1971).

[3] M. Doi and S. F. Edwards, The Theory of Polymer Dynamics (Oxford Science Publications, New York, 1990).

[4] T. C. B. McLeish, Adv. Phys. 51, 1379 (2002).

[5] C. Bennemann, J. Baschnagel, W. Paul, and K. Binder, Comput. Theor. Polym. Sci. 9, 217 (1999).

[6] A. J. Moreno and J. Colmenero, Phys. Rev. Lett. 100, 126001 (2008).

[7] M. Doxastakis, D. N. Theodorou, G. Fytas, F. Kremer, R. Faller, F. Müller-Plathe, and N. Hadjichristidis, J. Chem. Phys. 119, 6883 (2003).

[8] M. Brodeck, F. Alvarez, A. J. Moreno, J. Colmenero, and D. Richter, Macromolecules 43, 3036 (2010).

[9] S. Arrese-Igor, A. Alegría, A. J. Moreno, and J. Colmenero, Macromolecules 44, 3611 (2011).

[10] Thermally Stimulated Relaxations in Solids, Topics in Applied Physics, edited by P. Braünlich (Springer-Verlag, New York, 1979).

[11] Electrects, Topics in Applied Physics Vol. 33, edited by G. M. Sessler (Springer-Verlag, New York, 1980).

[12] V. Halpern, J. Phys. D 27, 2628 (1994).

[13] E. Dargen, M. Kattan, C. Cabot, P. Lebaudy, J. Ledru, and J. J. Grenet, J. Appl. Polym. Sci. 74, 2716 (1999).

[14] E. Laredo, M. Grimau, A. Müller, A. Bello, and N. Suarez, J. Polym. Sci. B 34, 2863 (1996).

[15] A. Alegría, L. Goitiandia, and J. Colmenero, J. Polym. Sci. B 38, 2105 (2000).

[16] D. Boese and F. Kermer, Macromolecules 23, 829 (1990).
[17] Y. Matsumiya, K. Kumazawa, M. Nagao, O. Urakawa, and H. Watanabe, Macromolecules 46, 6067 (2013).

[18] H. Watanabe, Y. Matsumiya, K. Osaki, and M. L. Yao, Macromolecules 31, 7528 (1998).

[19] As a general criteria, we have checked that using a $T_{\text {off }}$ temperature higher than the position of the maxima of the $p=3$ mode and preferably (but not necessarily) lower than the maxima of the $p=1$ mode is a good compromise to isolate the first and most intense mode. These temperatures can be easily estimated from global TSDC measurements without the need of external inputs.

[20] C. Bucci and R. Fieschi, Phys. Rev. Lett. 12, 16 (1964).

[21] C. Riedel, A. Alegría, P. Tordjeman, and J. Colmenero, Macromolecules 42, 8492 (2009).

[22] I. M. Hodge, J. Non-Cryst. Solids 169, 211 (1994).

[23] R. Kohlrausch, Pogg. Ann. Phys. Chem. 91, 179 (1854).

[24] G. Williams and D. C. Watts, Trans. Faraday Soc. 66, 80 (1970)

[25] L. Boltzmann, Pogg. Ann. Phys. 7, 624 (1876).

[26] At $M_{w} \lesssim 10000$ Da PI's $T_{g}$ depends on $M_{w}$ [21] and the "universal" WLF law describing the chain dynamics of higher $M_{w}$ PIs is no longer valid (even if actual $T_{g}$ is taken into account). Thus, in order to generate model current curves, particular WLF laws have been used for each low $M_{w}$ PI. In order to determine these particular WLF laws with high accuracy, BDS measurements were complemented with time domain isothermal measurements extending the covered frequency range up to $\sim 10^{4} \mathrm{~Hz}$.

[27] J. Colmenero, Macromolecules 46, 5363 (2013).

[28] In this case, we have considered $\tau_{\alpha}$ as the time scale of the corresponding incoherent scattering function (ISF) at momentum transfer $Q \sim 1 \AA^{-1}$. It is well documented that relaxation times from ISF at this $Q$ range generally match the $\alpha$-relaxation times determined by dielectric spectroscopy [see, e.g., J. Colmenero, A. Arbe, and A. Alegría, J. Non-Cryst. Solids 172-174, 126 (1994)].

[29] M. Brodeck, F. Alvarez, A. Arbe, F. Juranyi, T. Unruh, O. Holderer, J. Colmenero, and D. Richter, J. Chem. Phys. 130, 094908 (2009). 